\title{
金属有机框架抗菌材料的研究进展
}

\author{
齐野 $a, b$ 任双颂 ${ }^{c}$ 车颖 ${ }^{c}$ 叶俊伟*, $a, b$ 宁桂玲*,,$a$ \\ $\left({ }^{a}\right.$ 大连理工大学化工学院 精细化工国家重点实验室 大连 116024) \\ ( ${ }^{b}$ 辽宁省硼镁特种功能材料制备与应用技术工程实验室 大连 116024) \\ ( ${ }^{c}$ 大连医科大学附属第一医院 大连 116011)
}

\begin{abstract}
摘要 细菌耐药问题已经成为了中国乃至全球的重大公共健康威胁, 设计合成新型抗菌材料以减少抗生素依赖成为当 前化学化工、材料和生物医学领域中的重要研究课题. 金属有机框架(Metal-organic frameworks, MOFs)材料是由有机配 体和金属离子或团簇通过配位键自组装形成的多孔晶态材料, 在气体吸附与分离、传感和催化等领域都扮演着重要角 色. 为了寻求更好应对细菌威胁的方式方法, 国内外研究者们纷纷构建出不同结构的 MOFs 材料, 并将其应用于抗菌 领域. 本综述从细菌耐药性的产生和 MOFs 抗菌机理等方面出发, 分类概述了不同金属中心和配体 MOFs 材料、MOFs 包覆金属纳米粒子材料和药物缓释 MOFs 材料等在抗菌、促进伤口愈合等方面的应用, 归纳概括了 MOFs 材料在抗菌 领域应用中仍需解决的科学问题, 并对该领域的发展趋势进行了展望.
\end{abstract}

关键词＼cjkstart金属有机框架; 抗菌; 机理; 活性氧自由基; 药物输送

\section{Research Progress of Metal-Organic Frameworks Based Antibacterial Materials}

\author{
Qi, Ye $\mathrm{R}^{a, b}$ Ren, Shuangsong ${ }^{c} \quad$ Che, Ying $^{c} \quad$ Ye, Junwei*,a,b Ning, Guiling*,a,b \\ ( ${ }^{a}$ State Key Laboratory of Fine Chemicals, School of Chemical Engineering, Dalian University of Technology, \\ Dalian 116024) \\ $\left({ }^{b}\right.$ Engineering Laboratory of Boric and Magnesic Functional Material Preparative and Applied Technology in Liaoning \\ Province, Dalian 116024) \\ ( ${ }^{c}$ The First Affiliated Hospital of Dalian Medical University, Dalian 116011)
}

\begin{abstract}
With the accelerating process of industrialization and urbanization, as well as the increasing proportion of the elderly in the world's population, we are facing more complex health threats related to bacterial infection. While the vast majority of the bacteria in the body are rendered harmless by the protective effects of the immune system, the continued abuse and misuse of antibiotics has accelerated the spread of antibiotic-resistant bacterial strains and has resulted in substantial new challenges with respect to modern-day antibiotic-based treatments. Therefore, intelligent design of new antibacterial modalities to be used for treating human and livestock diseases is an extremely urgent priority for researchers in the fields of chemistry, chemical engineering, materials and biomedical sciences. Toward this end, the most intriguing of the new developments are metal-organic frameworks (MOFs). MOFs are versatile crystalline porous lattices of organic ligands and metal ion/clusters that formed by self-assembly via coordination bonds. Due to their unique characteristics, including relatively straight forward and simple methods for synthesis, large surface areas, novel and diverse structures, and adjustable porosity, MOFs not only play strong roles with respect to novel methods for gas storage and separation, they may also be utilized in unique applications associated with sensors mechanisms and catalysis. These features contribute to our current understanding of MOFs as promising candidates for the development of pharmaceutical and specifically antibacterial applications. In this review, antibacterial mechanisms, and the development of resistance to current antibiotic strategies are summarized and discussed. The main mechanisms by which bacteria show resistance to antibiotics include altered metabolic pathways, regulation of target sites, and inactivation, modification, and/or reduction in the capacity to accumulate antibacterial drugs. We consider recent progress on the development of MOFs, including the use of specific metal centers and ligands, metal nanoparticles, and drug-encapsulation, all of which have important applications with respect to antibacterial activities, and wound healing. Finally, the challenges and prospects of MOF-based antibacterial materials are discussed, including critical findings, which will help toward the development of the next generation antibacterial MOFs for human use.
\end{abstract}

Keywords metal-organic framework; antibacterial; mechanism; reactive oxygen species; drug delivery

* E-mail: junweiye@dlut.edu.cn; ningg1@dlut.edu.cn

Received April 28, 2020; published May 28, 2020.

Project supported by the National Natural Science Foundation of China (Nos. U1808210, U1607101) and the Fundamental Research Funds for the Central Universities (No. DUT20LK37).

项目受国家自然科学基金(Nos. U1808210, U1607101)和中央高校基本科研业务费(No. DUT20LK37)资助. 


\section{1 引言}

近年来, 耐药菌感染持续增多, 特别是治疗手段有 限的多重耐药菌和广泛耐药菌的感染发生率不断提高, 导致部分患者病程延长、住院时间延长并承担着更高的 死亡风险, 加重了家庭负担 ${ }^{[1,2]}$. 细菌耐药问题已经构成 了我国乃至全球的重大公共健康威胁 ${ }^{[3]}$. 因此研制高效 抗菌抗感染新型功能材料对于保护伤口、治疗耐药细菌 感染及提高患者生存率具有重要战略意义 ${ }^{[4]}$. 目前在临 床上可供选择的抗菌药物数量极为有限, 最后极可能需 要使用具有潜在毒性的抗菌方案. 例如, 对耐碳青霉烯 类的革兰氏阴性菌感染, 给予持续大剂量多粘菌素或氨 基糖苷类抗生素抗感染治疗, 这可导致急性肾损伤的发 生, 特别是对原来就存在肾功能不全、休克或需同时使 用其它肾毒性药物的患者, 损伤作用更为显著. 此外, 超大剂量使用碳青霉烯类和头孢吡肜等抗菌药物, 可能 会带来更严重的健康损害. 当前, 传统抗菌剂主要分为 季铵盐类等有机抗菌剂、壳聚糖等天然抗菌剂、金属离 子及其单质和 $\mathrm{TiO}_{2} 、 \mathrm{CeO}_{2}$ 等氧化物为代表的无机抗菌 剂 ${ }^{[5-8]}$. 但是这些抗菌剂往往存在着耐热性差、规模化生 产较难、金属毒性较大及必须在紫外线照射和氧气(或 水)的存在下才能起到杀菌作用等缺点. 如何结合各类 抗菌剂的优点、摒弃其缺点并提高抗菌活性成为了摆在 科研工作者面前的一道难题 $[9,10]$.

金属有机框架材料 (Metal-organic frameworks, $\mathrm{MOF}$ ) 是一类发展十分迅速的有机一无机杂化材料, 可 按需形成一维(1D)、二维(2D)或三维(3D)空间结构, 近 年来受到越来越多的关注 ${ }^{[11-14]}$. MOFs 可通过调节不同 金属节点和有机配体来实现拓扑结构的多样化, 并且其 孔径范围从微孔、中孔到大孔都可以任意调控. 由于结 构的多样性、可设计性、可剪裁性以及超高的比表面积、 良好的生物相容性和生物降解性等卓越的理化性质, MOFs 在包括催化、传感检测、癌症治疗和抗菌等诸多 领域中具有广阔的应用前景 ${ }^{[15-21]}$. 特别是 MOFs 的出现, 更加有力地整合了多种具有潜在抗菌应用的材料 ${ }^{[22-24]}$. 基于以下几方面特征, 科研工作者们将 MOFs 视为具有 良好应用前景的第三代新型抗菌剂 ${ }^{[25,26]}$ : (1) MOFs 中的 金属节点(如 $\mathrm{Ag} 、 \mathrm{Cu} 、 \mathrm{Ni} 、 \mathrm{Zn}$ 等贵金属和过渡金属)具 有持续释放金属离子的特性; (2)有机多配位配体(如卟 啉衍生物、咪唑盐等)具有良好的抗菌活性和生物相容 性; (3)在某些情况下, 孔道结构在消除客体分子的过程 中十分稳定, 较高的比表面积和大的孔隙率有利于金属 纳米粒子(M-NP)、生物大分子等抗菌活性物质的有效封 装和输送; (4)在生物体内具有足够的稳定性和较低的生 物毒性, 在执行其特定功能后可有效生物降解.

本文综述了细菌耐药性的产生机理, 重点介绍了近 三年来不同金属中心和配体 MOFs 材料、MOFs 包覆金 属纳米粒子材料和药物缓释 MOFs 材料(图 1)等在抗菌、 抗感染和生态环境保护等方面的最新研究进展, 特别是
国内科研工作者取得的优秀研究成果，阐明了 MOFs 抗 菌材料当前存在的关键科学问题和应用问题, 并对研究 发展趋势进行了展望.

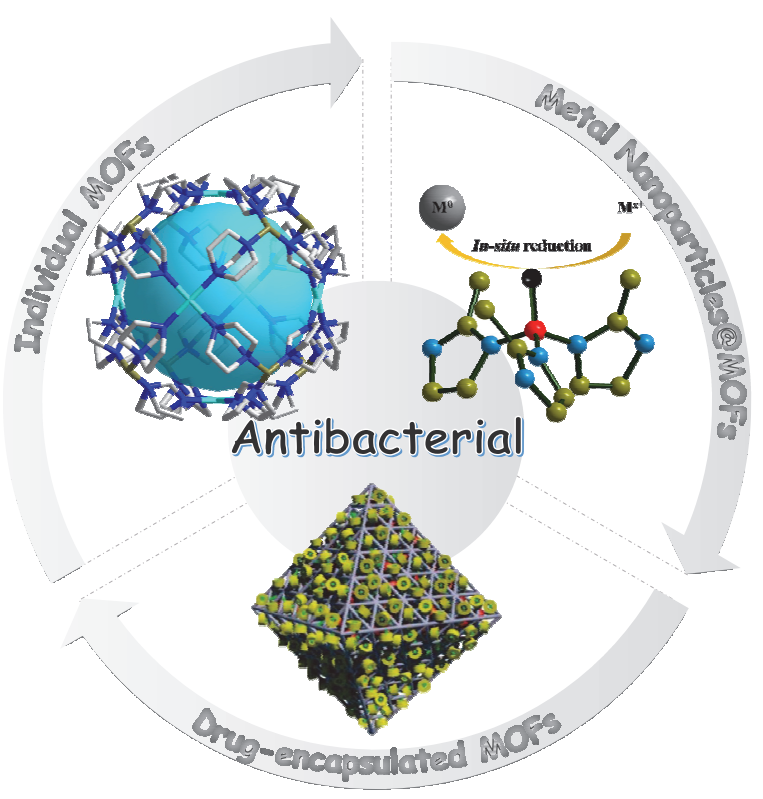

图 1 三种典型的 MOFs 材料在抗菌领域中的应用

Figure 1 Three typical metal-organic frameworks for antibacterial

\section{2 细菌对传统抗生素的耐药性产生机理}

自 Alexander ${ }^{[27]}$ 发现并分离出青霉素以来, 抗生素 治疗法就变成了对抗细菌感染最强有力的武器. 抗生素 之所以能够有效地杀灭细菌, 主要是它能够抑制细菌细 胞壁的合成、抑制细菌关键蛋白质的合成、抑制 DNA 的合成及抑制细菌繁殖与生长 ${ }^{[28,29]}$. 近年来, 由于抗生 素的使用越来越普遍，特别是在部分发展中国家，无需 医师处方即可购买抗生素; 并且抗生素随着养殖废水和 医疗废水等途径进入水体. 这些因素均导致抗生素耐药 性的不断增加. 据统计, 目前我国抗生素消耗量达 20 万 吨, 约占全球消耗量的一半 ${ }^{[30]}$. 由于抗生素无节制的使 用及滥用, 目前已经出现了许多的多重耐药 “超级细 菌” [31,32]. 例如, 金黄色葡萄球菌(Staphylococcus aureus, S. aureus) 是一种自然界普遍存在的细菌, 常寄生于人和 动物的皮肤、鼻腔和肠胃等组织器官.一般情况下, 因 为人体免疫系统的保护作用, S. aureus 并不会损害人体 健康, 通常只在皮肤伤口处才会造成细菌感染, 但由于 抗生素无节制的使用, S. aureus 的菌株已产生抗药性. 如若人体免疫系统出现问题, 即使没有伤口亦可导致耐 甲氧西林金黄色葡萄球菌(Methicillin-resistant S. aureus, MRSA)感染. 临床上 MRSA 感染一般会使用万古霉素 (Vancomycin, Van)来治疗, 不过临床上已经发现 Van 的 疗效有所下降.

细菌耐药性究竟是如何产生的? 微生物学家一直 
在不停探索这个问题的答案. 其实抗性细菌的出现甚至 早于青霉素的发现. 1943 年, Luria 和 Delbruck ${ }^{[33]}$ 发现了 细菌具有 “主动进化” 现象, 即面对噬菌体感染, 细菌 为了存活下去, 自发地进行随机突变以逃避噬菌体的侵 袭. 一般地, 对于一切生命体而言, 在环境适宜的外界 条件下, 发生基因突变的频率是非常低的. 但是, 科学 家已经发现由于抗生素的广泛使用, 细菌正通过 “进化 压力” 机制来增加耐药性 ${ }^{[34,35]}$. 这种 “进化压力” 促进 了遗传多样性, 提高了细菌对环境的适应能力和耐药能 力 $^{[36]}$.

细菌耐药性产生机理如图 2 所示 ${ }^{[37-39]}$ : (1)改变代谢 途径. 细菌通过加强对细胞壁的修饰抑制抗生素摄取. 例如, 叶酸是合成细胞壁的主要原料之一, 磺酰胺类药 物通过破坏叶酸合成中间体对氨基苯甲酸来抑制细菌 繁殖, 而耐药性细菌和哺乳动物细胞一样, 会使用预先 合成的叶酸增强细胞壁; (2)改变靶位点或结合位点. 细 菌会主动修饰药物靶标, 使药物无法发挥药效. 例如, MRSA 以及其他类型的耐青霉素细菌可以通过改变青 霉素的结合位点来避免抗生素与细胞核糖体结合, 使得 核糖体可以继续合成细胞所必需的蛋白质. 在革兰氏阴 性细菌中, 质粒 (Plasmid) 介导抗性基因产生可以与 DNA 促旋酶相结合的蛋白质, 这种蛋白质可以降低 DNA 促旋酶与喹诺酮类抗生素之间的亲和力, 从而降 低喹诺酮的药物有效性; (3)灭活或修饰抗生素. 合成药 物失活酶, 引发药物失活. 例如, 抗青霉素细菌通过产 生 $\beta$-内酰胺酶促使青霉素 $\mathrm{G}$ 失活. 另外, 细菌产生的保 护酶可以在抗生素的特定位点添加一个乙酰基或磷酸 基团, 进而降低抗生素与细菌核糖体结合的能力; (4)细 菌主动外排系统的过度表达. 激活外排㫤, 主动减少药 物蓄积. 例如, 抗氟喹诺酮细菌通过降低药物的渗透性 或是激活细胞膜表面上的外排百, 将抗生素主动排出. 细菌外排泵的出现通常与抗生素特定底物有关, 这种主 动外排过程并不会对细胞造成任何实质损伤.

滥用抗生素的危害还不仅仅是使细菌产生抗药性, 抗生素在局部给药过程中往往难以确定其使用量, 使用 量过大可能在患处发生超敏反应或接触性皮炎 ${ }^{[40]}$. 人 体大部分的免疫功能是以肠道中菌群平衡为基础. 抗生 素长期使用杀灭大量益生菌, 破坏肠道微生态平衡, 为 体外病菌侵入繁殖创造了条件. 当前, 抗生素已经无法 满足对抗多重耐药菌感染的需要, 因此其他新型抗菌方 法逐渐被使用起来, 例如, 氯气等强氧化杀菌剂、低温 等离子体等方法和技术 ${ }^{[41,42]}$. 然而, 这些方法或技术由 于自身的强腐蚀性、对环境的二次污染、对设备或技术 要求高等不利因素, 限制了它们在生态环境和生物体内 的抗菌应用. 因此, 迫切需要研发出能高效杀菌、避免 耐药性产生的新型抗菌药剂以减少对抗生素的依赖. MOFs 与传统抗生素在抗菌机理上有着本质性的不同, 从而避免了细菌耐药性的产生.

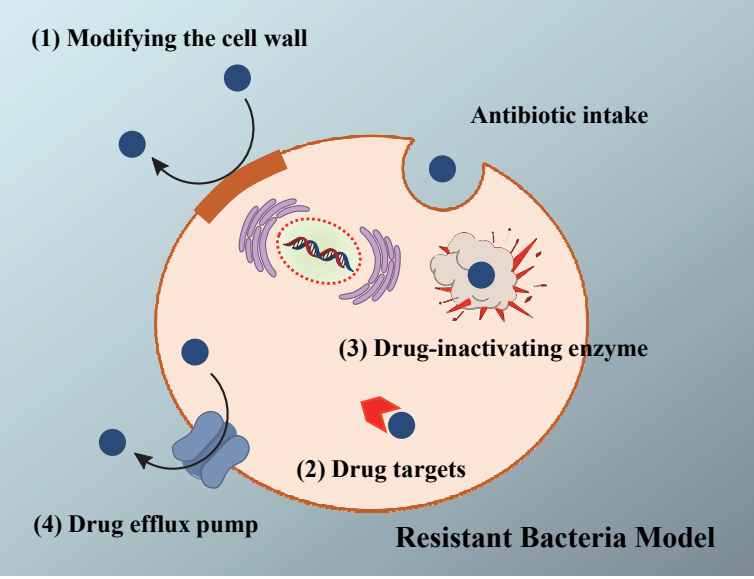

图 2 细菌耐药性产生机理示意图 ${ }^{[37-39]}$

Figure 2 Schematic illustration of bacterial resistance mechanism ${ }^{[37-39]}$

\section{3 不同金属中心和配体 MOFs 抗菌材料}

MOFs 可以看作是金属离子的储存库，具有抗菌功 能的银 $\left(\mathrm{Ag}^{+}\right)$、锌 $\left(\mathrm{Zn}^{2+}\right)$ 、铜 $\left(\mathrm{Cu}^{+} / \mathrm{Cu}^{2+}\right)$ 和钴 $\left(\mathrm{Co}^{2+}\right)$ 等离子 可通过 MOFs 降解过程而逐渐释放以达到持久抗菌效 果. 此外, MOFs 结构中同时存在的有机配体也对抗菌 作用的产生起着积极的作用. 不同金属中心和配体 MOFs 材料的抗菌机理有以下几种(图 3a): (1)改变细胞 膜通透性. 金属离子通过改变细胞膜的通透性，使细胞 内营养物质等内容物泄露, 导致细胞死亡; (2)损伤 DNA. MOFs 释放的金属离子或有机配体可以破坏 DNA 或蛋白酶，进而使 DNA 分子发生交联，结构被破坏，造 成细胞死亡或丧失分裂增殖的能力; (3)降低脱氢酶活 性. 金属离子可与细胞蛋白质中的颈基、氨基等基团发 生反应，破坏细胞的电子传输系统、呼吸系统和物质传 输系统; (4)氧化应激反应. 部分金属离子或羧酸类、咪 唑类和卟啉类等有机配体可诱导细胞产生大量的活性 氧自由基团(Reactive oxygen species, ROS), 进一步造成 细菌细胞内还原型辅酶 II (NADPH)氧化酶类抑制剂 (DPI)含量的减少, 以加速细菌死亡. 值得一提的是, 在 多因素协同作用下，细菌可被有效杀灭且不会产生耐药 性. 近年来, 许多科研人员为了寻找新型 MOFs 抗菌材 料做出了努力, 也取得了一些重要研究成果(表 1 ). 接下 来, 我们分类阐述不同金属离子和配体 MOFs 抗菌材料 的最新研究进展.

$\mathrm{Ag}^{+}$是一个既传统又经常被推陈出新的抗菌 剂 ${ }^{[43-45]}$. 古代的勋贵人家使用银质器皿来盛装食物以防 止食物腐败变质; 银质的耳钉可以防止伤口感染; 直到 今天, 东南亚部分国家的僧侣依然使用银碗来化缘. 科 研人员也正是从中获得启发, 研制出了各种类型的银离 子抗菌产品. Jaffres 及其同事 ${ }^{[46]}$ 在 2011 年报告了一种 $3 \mathrm{D} A g-\mathrm{MOF}$, 它由 $\mathrm{Ag}^{+}$和同时带有羧基和膦酸官能团的 3-膦酰基苯甲酸所构成, 并通过 $\mathrm{Ag}^{+}$的长效缓释来有效 杀灭包括 MRSA、大肠杆菌(Escherichia coli, E. coli) 
MG1655 菌株和铜绿假单胞菌(Pseudomonas aeruginosa, P. aeruginosa) 在内的 6 种细菌. 而该 Ag-MOF 对人红细 胞的毒性极其微弱. 迄今为止, 以羧酸盐为有机配体的 Ag-MOF 最受关注, 主要原因是金属-羧酸盐配位键的 形成有助于形成高度有序的晶体结构, 且具有一定的抗 菌性; 羧酸基团的双齿双桥配位能力有利于从孔道中抽 出溶剂, 保持稳定的骨架结构. 2014 年起, 宁桂玲团 队 ${ }^{[47-49]}$ 先后利用 3-硝基邻苯二甲酸、5-硝基间苯二甲酸、 均苯三甲酸和对苯二羧酸等多种有机翔酸配体, 合成了 一系列含有单核/多核建构单元的 Ag-MOF. 最近, 其团 队通过静电纺丝法成功合成了 $\mathrm{Ag}_{2}$ [HBTC][im]聚乳酸 (Ag-MOF-PLA)复合纤维膜(图 3b) ${ }^{[50]}$. 该复合纤维膜对 E. coli, P. aeruginosa, S. aureus, 耻垢分枝杆菌(Mycobacterium smegmatis, M. smegmatis)等 4 种细菌的灭活率 均超过 $95.0 \%$. 他们又构建了 S. aureus 感染的急性全层 皮肤组织损伤小鼠模型, $14 \mathrm{~d}$ 内治疗组的伤口愈合率为 99.9\%. 通过 $\mathrm{Ag}^{+}$释放实验和蛋白质组学分析, 抗菌活 性的产生主要归因于 $\mathrm{Ag}^{+}$的缓慢释放和 ROS 的协同作 用, $\mathrm{Ag}^{+}$和 ROS 共同破坏了细胞壁和质膜的能量代谢以 及细胞的物质传输系统.

$\mathrm{Zn}^{2+}$ 是一种无毒的抗菌性阳离子, 被广泛用于皮肤 科作为收敛剂、保湿剂及抗菌剂 ${ }^{[51,52]}$. 一些研究发现, 基于咪唑配体的 Zn-MOFs 对 E. coli, P. aeruginosa, $S$.

(a) Antibacterial mechanism

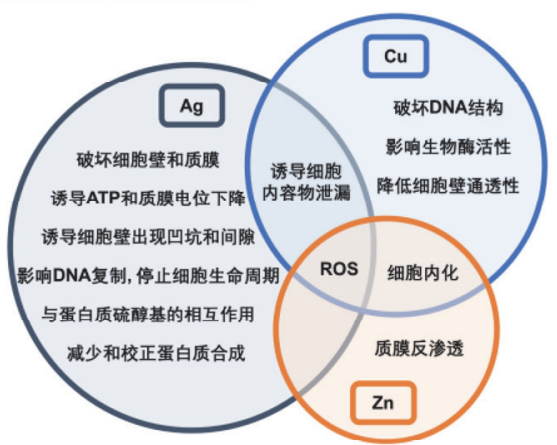

(c) Zn-imidazole-MOFs

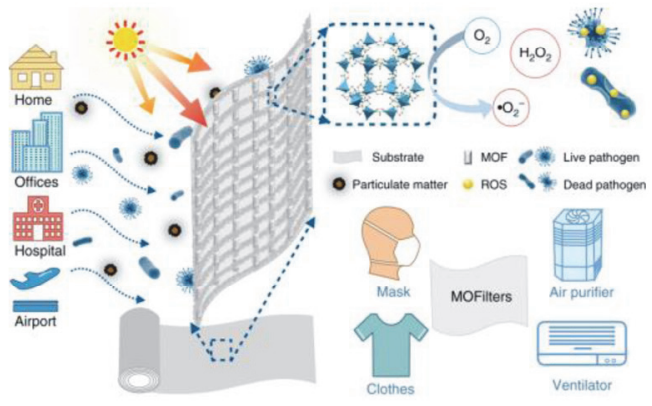

aureus 和 B. subtilis 等具有较强的抗菌活性. 更有趣的 是，近年来被广泛研究的 ZIF-8 (由 $\mathrm{Zn}^{2+}$ 和 2-甲基咪唑构 成的沸石咪唑酯骨架结构材料)具有产生 ROS 的能力. 2019 年, 王博团队 ${ }^{[53]}$ 结合前期的研究工作制备了一系 列具有光催化活性的 MOFs, 笁选了具有超高光催化杀 菌活性的 ZIF-8, 实现对水体中 E. coli 的高效杀灭, 杀 菌率大于 99.9999\%(图 3c). 然后利用热压法设计合成了 新型的高效综合空气过滤器(MOFilter), PM 微粒捕获效 率能达到 $98 \%$ 以上，同时还可以杀灭空气中 $99.99 \%$ 的 细菌. 机理研究发现, ZIF-8 在太阳光催化作用下, 通过 配体与金属的电荷转移产生光生电子, 并且光生电子可 以有效地活化 $\mathrm{O}_{2}$ 形成・ $\mathrm{O}^{2-}$ 和 $\mathrm{H}_{2} \mathrm{O}_{2}$, 使空气中的致病细 菌氧化致死. 这项工作为光催化抗菌 MOFs 的规模生产 打下了坚实的基础.

$\mathrm{Cu}$ 是生物体保持生命活性的必需微量元素之一， 同时也是投入实际应用最早的一批长效抗菌材料 ${ }^{[54,55]}$. Rauf 等 ${ }^{[56]}$ 发现 $\mathrm{Cu}^{2+}$ 和噻吩二羧酸配体等组成的 $\mathrm{Cu}-\mathrm{MOF}$ 对 $S$. aureus 的生长具有较强的抑制作用. 与 $\mathrm{Ag}-\mathrm{MOF}$ 抗菌机理类似, 以 $\mathrm{Cu}^{2+}$ 为金属中心的 MOFs 材 料向环境中缓慢释放 $\mathrm{Cu}^{2+}$ 以破坏细菌结构. 同时, 配体 官能团与细胞蛋白的氢键作用可以增加材料的细胞脂 溶性, 帮助 $\mathrm{Cu}^{2+}$ 穿透细胞表层, 也提高了这类材料的抑 菌活性. 值得注意的是, $\mathrm{Cu}-\mathrm{MOF}$ 也产生了大量的羟

(b) Ag-carboxylic acid-MOFs

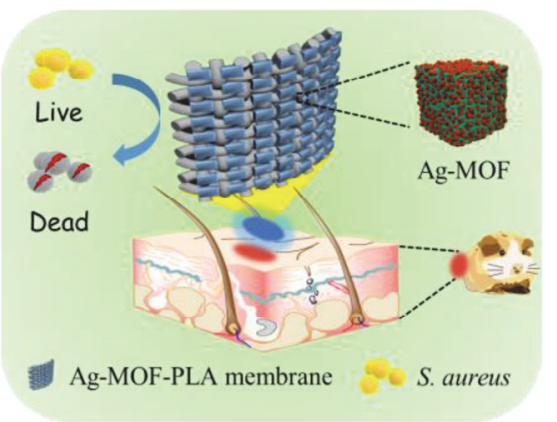

(d) Cu-porphyrin-MOFs

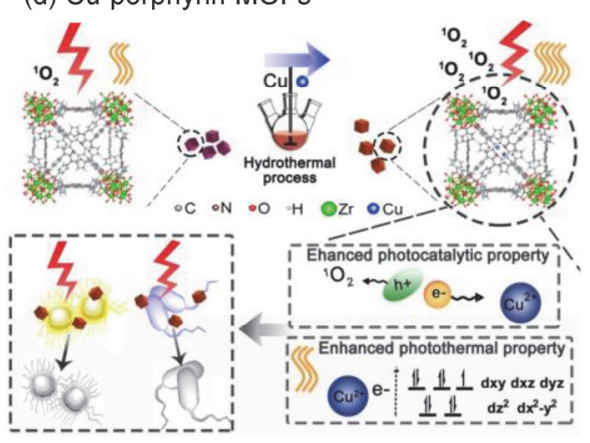

图 3 (a) 不同金属离子的抗菌机理示意图、(b)用于治疗细菌感染小鼠皮肤伤口的 Ag-MOF-PLA 复合膜敷料 ${ }^{[50]}$ (c)基于 ZIF-8 的 MOFilter 在空 气污染防治和个人卫生防护中的高效灭菌应用 ${ }^{[53]}$ 和 $(\mathrm{d}) \mathrm{Cu}^{2+}$ 掺杂的金属卟啉-MOF 制备方法、晶体结构、结构成分以及抗菌机理 ${ }^{[57]}$

Figure 3 (a) Schematic illustrating the specific antibacterial mechanisms of particular metals, (b) Ag-MOF-PLA antibacterial applications for wound dressing through the mouse skin wound model ${ }^{[50]}$ (Copyright 2020, Elsevier), (c) application of MOFilter based on ZIF-8 for photocatalytic high efficiency sterilization in air pollution and personal protection ${ }^{[53]}$ (Copyright 2019, Springer Nature) and (d) preparation procedures of different kinds of MOFs, as well as the crystal structure, structural components, and antibacterial mechanism of the MOFs obtained ${ }^{[57]}$ (Copyright 2020, Elsevier) 
表 1 不同金属中心 MOFs 材料的抗菌性质 ${ }^{a}$

Table 1 Antibacterial properties of typical different metal centers-constructed MOFs

\begin{tabular}{|c|c|c|c|c|}
\hline $\begin{array}{l}\text { 中心金属 } \\
\text { 离子 }\end{array}$ & 化合物 & 抗菌性能 & 抗菌机理 & Ref. \\
\hline \multirow{6}{*}{$\mathrm{Ag}^{+}$} & $\mathrm{Ag}_{2}(\mathrm{O}-\mathrm{IPA})\left(\mathrm{H}_{2} \mathrm{O}\right) \cdot\left(\mathrm{H}_{3} \mathrm{O}\right)$ & E. coli: $\mathrm{MIC}=5 \sim 10 \mu \mathrm{g} / \mathrm{mL} ;$ S. aureus: $\mathrm{MIC}=10 \sim 15 \mu \mathrm{g} / \mathrm{mL}$ & $\mathrm{Ag}^{+}$释放; 破坏细胞壁; 灭活生物酶 & [58] \\
\hline & $\mathrm{Ag}_{3}(3$-phosphonobenzoate) & $\begin{array}{l}\text { E. coli: } \mathrm{MBC}=50 \mathrm{mmol} / \mathrm{L} ; \text { S. aureus: } \mathrm{MBC}=50 \sim 75 \mathrm{mmol} / \mathrm{L} ; \\
\text { P. aeruginosa: } \mathrm{MBC}=20 \sim 30 \mathrm{mmol} / \mathrm{L}\end{array}$ & $\mathrm{Ag}^{+}$释放 & [46] \\
\hline & {$\left[(\mathrm{AgL}) \mathrm{NO}_{3}\right] \cdot 2 \mathrm{H}_{2} \mathrm{O}$} & E. coli: $\mathrm{MIC}=300 \mu \mathrm{g} / \mathrm{mL} ;$ S. aureus: $\mathrm{MIC}=297 \mu \mathrm{g} / \mathrm{mL}$ & $\mathrm{Ag}^{+}$释放 & [59] \\
\hline & $\operatorname{Ag}(\operatorname{Bim})$ & E. coli: $\mathrm{MIC}=10 \sim 15 \mu \mathrm{g} / \mathrm{mL} ;$ S. aureus: $\mathrm{MIC}=15 \sim 20 \mu \mathrm{g} / \mathrm{mL}$ & $\mathrm{Ag}^{+}$释放 & \multirow{2}{*}{ [47] } \\
\hline & $\mathrm{Ag}_{6}(4-\mathrm{NPTA})(\mathrm{Bim})_{4}$ & E. coli $: \mathrm{MIC}=5 \sim 10 \mu \mathrm{g} / \mathrm{mL} ;$ S. aureus: $\mathrm{MIC}=10 \sim 15 \mu \mathrm{g} / \mathrm{mL}$ & $\mathrm{Ag}^{+}$释放 & \\
\hline & {$\left[\mathrm{Ag}\left(\mathrm{NO}_{3}\right)\left(\mu_{3}-\mathrm{PTA}=\mathrm{O}\right)\right]_{n}$} & $\begin{array}{c}\text { E. coli: } \mathrm{MIC}=6 \mu \mathrm{g} / \mathrm{mL} ; \text { P. aeruginosa: } \mathrm{MIC}=7 \mu \mathrm{g} / \mathrm{mL} ; \text { S. aureus: } \\
\mathrm{MIC}=20 \mu \mathrm{g} / \mathrm{mL} ; \text { C. albicans: } \mathrm{MIC}=20 \mu \mathrm{g} / \mathrm{mL}\end{array}$ & $\mathrm{Ag}^{+}$释放 & [60] \\
\hline \multirow{6}{*}{$\mathrm{Zn}^{2+}$} & Zn-MOF & $\begin{array}{l}\text { S. aureus, E. coli, S. enterica, K. pneumoniae, B. subtilis, A. baumannii: } \\
\qquad \mathrm{MIC}=0.006 \mathrm{~g} / 100 \mathrm{cc}\end{array}$ & $\mathrm{Zn}^{2+}$ 与配体的释放 & [61] \\
\hline & GOZIF-8 & E. coli: 抑菌率 $=81.1 \% ;$ S. aureus: 抑菌率 $=85.7 \%$ & $\mathrm{Zn}^{2+}$ 释放; 咪唑配体; ROS & [62] \\
\hline & Cotton/ZIF-8 fabric & $\begin{array}{c}\text { E. coli: 平均菌落数 }=0 \mathrm{CFU} / \mathrm{cm}^{2} ; \text { S. aureus: 平均菌落数 }=0 \\
\qquad \mathrm{CFU} / \mathrm{cm}^{2}\end{array}$ & \multirow{3}{*}{$\begin{array}{c}\mathrm{Zn}^{2+} \text { 与细胞膜蛋白相互作用破坏了细 } \\
\text { 菌结构, 继而引发细胞内化 } \\
\mathrm{Zn}^{2+} \text { 的释放; 硼味唑配体破坏细菌脂 } \\
\text { 质体; } \mathrm{ROS} \\
\mathrm{Zn}^{2+} \text { 释放; 咪唑配体 }\end{array}$} & [63] \\
\hline & Zn-BIF & E. coli: $\mathrm{MIC}=500 \mu \mathrm{g} / \mathrm{mL} ;$ S. aureus: $\mathrm{MIC}=500 \mu \mathrm{g} / \mathrm{mL}$ & & {$[64]$} \\
\hline & FZIF-8 & E. coli: 抑菌圈直径 $=5.44 \mathrm{~mm}$; S. aureus: 抑菌圈直径 $=22.1 \mathrm{~mm}$ & & [65] \\
\hline & $\mathrm{Zn}(\mathrm{II}) \mathrm{MOF}(1)$ & S. haemolyticus: $\mathrm{MIC}=270 \mu \mathrm{g} / \mathrm{mL} ;$ E. aerogenes: $\mathrm{MIC}=250 \mu \mathrm{g} / \mathrm{mL}$ & - & [66] \\
\hline \multirow{6}{*}{$\mathrm{Cu}^{2+}$} & AC-HKUST-1 & P. aeruginosa: $\mathrm{MIC}=50 \mu \mathrm{g} / \mathrm{mL} ; \mathrm{MBC}=100 \mu \mathrm{g} / \mathrm{mL}$ & $\mathrm{Cu}^{2+}$ 释放 & [67] \\
\hline & CuBTC-silk fiber & E. coli: 抑菌圈直径 $=10 \mathrm{~mm} ;$ S. aureus: 抑菌圈直径 $=27 \mathrm{~mm}$. & $\mathrm{Cu}^{2+}$ 释放 $^{2}$ & [68] \\
\hline & {$\left[\mathrm{Cu}(\mathrm{ctp})\left(\mathrm{H}_{2} \mathrm{O}\right)_{2}\right]_{n}$} & $\begin{array}{c}\text { B. subtilis: } \mathrm{MIC}=5 \sim 10 \mu \mathrm{g} / \mathrm{mL} ; \text { S. aureus: } \mathrm{MIC}=5 \sim 10 \mu \mathrm{g} / \mathrm{mL} ; S . \\
\text { enteritidis: } \mathrm{MIC}=5 \sim 10 \mu \mathrm{g} / \mathrm{mL}\end{array}$ & $\begin{array}{c}\mathrm{Cu}^{2+} \text { 释放; 利用中心原子的亲脂性, 有 } \\
\text { 利于渗透细胞膜脂质层 }\end{array}$ & [69] \\
\hline & {$\left[\mathrm{Cu}\left(\mu_{2}-\mathrm{Amp}\right)_{2}\right]_{n}$} & E. coli: 抑菌圈直径 $=15 \mathrm{~mm}$; S. aureus: 抑菌圈直径 $=12 \mathrm{~mm}$ & $\mathrm{Cu}^{2+}$ 释放 & [70] \\
\hline & $\mathrm{Cu}-\mathrm{H}_{2}$ bpdc-Gu & P. aeruginosa: $\mathrm{MIC}=500 \mu \mathrm{g} / \mathrm{mL} ; \mathrm{MBC}=700 \mu \mathrm{g} / \mathrm{mL}$ & $\mathrm{Cu}^{2+}$ 释放 & {$[71]$} \\
\hline & {$\left[\mathrm{Cu}(\mathrm{TDC})\left(\mathrm{H}_{2} \mathrm{O}\right)_{2}\right] \cdot \mathrm{H}_{2} \mathrm{O}$} & E. coli: $\mathrm{MIC}=150 \sim 200 \mu \mathrm{g} / \mathrm{mL} ;$ S. aureus: $\mathrm{MIC}=150 \sim 200 \mu \mathrm{g} / \mathrm{mL}$ & $\mathrm{Cu}^{2+}$ 释放; ROS & [56] \\
\hline
\end{tabular}

$a$ 最小抑菌浓度(Minimum Inhibitory Concentration, MIC), 最小杀菌浓度(Minimum Bactericidal Concentration, MBC), 白色念珠菌(Candida albicans, C. albicans), 肠炎沙门氏菌(Salmonella enteritidis, S. enteritidis), 肺炎克雷伯氏菌(Klebsiella pneumoniae, K. pneumoniae), 枯草芽孢杆菌(Bacillus subtilis, B. subtilis), 鲍曼不动杆菌(Acinetobacter baumannii, A. baumannii), 溶血葡萄球菌(Staphylococcus haemolyticus, S. haemolyticus)和产气大肠杆菌(Enterobacter aerogenes, E. aerogenes).

基自由基 $(\cdot \mathrm{OH})$, 并通过氧化损伤 DNA 以阻滞细胞周 期, 造成细胞死亡.

除了以上几种可以产生 ROS 的 MOFs 外，最为人熟 知的就是以卟啉类、酞菁类和卟吩类等光敏剂等为有机 配体的光动力型(Photodynamic therapy, PDT) MOFs 材 料 ${ }^{[72-74]}$. PDT 是利用光和光敏剂产生的光动力效应进行 疾病治疗的一种手段. PDT 可通过无毒的激发态光敏剂 耦合基态氧气产生单线态氧 $\left({ }^{1} \mathrm{O}_{2}\right)$, 引发细胞毒性, 促使 细菌细胞死亡. 近年的一些研究发现, PDT 对细菌的杀 灭效果十分显著. 例如, 2017 年, 谢志刚团队 ${ }^{[75]}$ 通过刮 涂法制备了含卟啉结构的 MOF-525 与聚 $\varepsilon$-己内酯的复 合膜, 在 LED 辐照下, 可对 E. coli 起到高效杀灭. 尽管 PDT 是一种有效的细菌感染治疗手段, 但也有以下的缺 点: (1)残留体内的光敏剂可造成光敏性副作用; (2)光的 穿透深度浅, 无法有效杀伤深处的细菌感染; (3)深部感 染通常是乏氧的, 进而使依赖于氧气的 PDT 疗效不佳. 不过, 光动力与其他抗菌方法联用可以弥补各自的不 足, 达到协同杀菌的目的. 2020 年, 吴水林团队 ${ }^{[57]}$ 通过 将适量的 $\mathrm{Cu}^{2+}$ 掺杂到吓啉中以形成金属卟啉 $\mathrm{MOFs}$ (图 3d). 这种 MOFs 具有优异的光催化性能, 其原理是掺杂 $\mathrm{Cu}^{2+}$ 的释放可以有效地捕获所产生的电子, 从而阻碍电
子-空穴的快速复合, 并最终提高了 MOFs 的光催化性 能. 此外, $\mathrm{Cu}^{2+}$ 也可以通过 $\mathrm{d}-\mathrm{d}$ 跃迁来增强材料的光热 转换性能. 伤口愈合实验证明, 通过热量、 ${ }^{1} \mathrm{O}_{2}$ 和 $\mathrm{Cu}^{2+}$ 的协同优化作用改变细胞的代谢途径，促进胶原蛋白沉 积并增强伤口修复能力，从而使该联合治疗技术成为当 前针对伤口细菌感染抗生素疗法的潜在替代性方案.

\section{MOFs 包覆金属纳米粒子抗菌材料}

以 $\mathrm{Ag}^{+}$或 $\mathrm{Cu}^{2+}$ 为配位中心的 $\mathrm{MOFs}$ 材料虽然通过离 子释放等途径获得优异的抗菌性能, 不过其生物安全性 和环境毒理性问题是必须加以考虑的 ${ }^{[76]}$. 大量的 $\mathrm{Ag}^{+}$进 入正常细胞的线粒体，可诱发机械性损伤，阻断电子在 呼吸链上的传递，最终破坏线粒体正常的生物功能; 大 量的 $\mathrm{Ag}^{+}$可取代蛋白分子中的 $\mathrm{Fe}^{2+}$, 后者则可通过芬顿 (Fenton)反应产生过量的 ROS，进而导致正常细胞发生 炎症反应; 大量的 $\mathrm{Ag}^{+}$排放到海洋环境中, 会严重抑制 硅藻细胞的生长、光合作用以及叶绿素的合成; $\mathrm{Ag}^{+}$在环 境中具有生物积累效应, 对水生生物有很强的毒性, 部 分海洋动物的生殖能力会受到抑制 ${ }^{[7]}$. 为了降低其副 作用, 通常可以使用银纳米粒子 $(\mathrm{AgNPs})$ 和铜纳米粒子 
(CuNPs)来替代 $\mathrm{Ag}^{+}$和 $\mathrm{Cu}^{2+} . \mathrm{M}-\mathrm{NP}$ 具有较高的比表面积 (其粒子尺寸通常小于 $10 \mathrm{~nm}$, 甚至是亚纳米级别), 可 以提供更多的活性位点与基质发生相互作用 ${ }^{[78]}$. 不过, M-NP 在溶液中通常具有较高的表面能, 从而通过范德 华力自发地团聚, M-NP 还面临易氧化和难回收等缺点. 因此, 在实践中最重要的是保持 M-NP 的稳定性、分散 性、高活性并确保其易回收. 目前, 完全清除 M-NP 和 寻找低毒替代品是减少环境风险最理想的方法 ${ }^{[79]}$. 此 外, 减少 M-NP 的暴露剂量和暴露时间也可以有效地减 轻对生态环境的危害. MOFs 的出现为解决这一问题提 供了新的策略.

MOFs 包覆金属纳米粒子(M-NP@MOFs)既保留了 MOFs 的高比表面积、大孔容和高孔隙率等优点, 又能 利用自身稳定、有序的孔道结构使 M-NP 均匀分散在孔 道中或表面上. M-NP@MOFs 在抗菌领域具有广阔的应 用前景, 这是因为: (1)与传统方法合成的 M-NP 相比, M-NP@MOFs 表面原子不饱和程度高, 表面缺陷多, 易 产生 ROS; (2) MOFs 利用自身孔道结构, 在限域空间效 应作用下, 合成的 M-NP 粒径均一且稳定性好; (3) M-NP 是通过配位键、 $\pi-\pi$ 键和范德华力等作用力与 MOFs 相结 合，可以进一步提高粒子的抗菌活性. M-NP@MOFs 的 合成方法主要分为 “瓶中造船” 法(ship-in-the-bottle)、“船 外造瓶” 法(bottle-around-a-ship)和 “原位还原”法(in-situ reduction)等三种.

“瓶中造船” 法, 即首先合成 MOFs, 再通过外加 $\mathrm{H}_{2} 、 \mathrm{CO}$ 和 $\mathrm{NaBH}_{4}$ 等还原性物质、固体研磨或紫外(UV) 光照还原等方法将 M-NP 装载到 MOFs 孔道中(图 $4 \mathrm{a})^{[80-82]}$. 2020 年, 倪永浩团队 ${ }^{[83]}$ 设计了一种多功能的 $3 \mathrm{D}$ 水凝胶, 该水凝胶由 AgNPs@MIL-100 和瓜尔豆胶 (GG)通过简单的共混合及后续自交联生成. 水凝胶作 为一种高含水量的多孔材料, 在细胞的 $3 \mathrm{D}$ 培养、组织 工程和伤口敷料等方面有着重要应用. 当前, 研制一种 具有高抗菌效率、良好生物相容性和机械强度的抗菌水 凝胶仍面临挑战. 他们通过 UV 光照还原金属前驱体来 合成 AgNPs, 不仅增强了 MIL-100 的光催化活性, 且赋 予了 $3 \mathrm{D}$ 水凝胶抗菌活性. 抗菌实验表明, 该水凝胶对 E. coli 的生长具有显著抑制作用. 此外, AgNPs@MIL100 与 $\mathrm{GG}$ 之间的复合还使得水凝胶具有可注射、易重 塑和自修复等特性，因而具有良好的应用前景.

“船外造瓶” 法, 即首先合成 M-NP, 然后将之与配 体一起加入到反应容器中进行后续反应(图 4b $)^{[84-86]}$. 一 般情况下, 预先合成的 M-NP 通常需要使用表面活性剂 改性以提高装配能力. 汪春昌课题组 ${ }^{[87]}$ 通过预先合成 $\mathrm{Ag}$ 纳米线并在其外层包覆 ZIF-8, 以形成贵金属 @MOFs 核壳异质结构. 通过改变反应时间等因素研究 了核壳结构的生长过程, 并且可以从技术上将 ZIF-8 壳 层厚度控制在 30 至 $100 \mathrm{~nm}$ 之间. 此外, 他们进一步测
试了Ag@ZIF-8 对 B. subtilis 和 E. coli BL21 的抗菌活性. 结果表明, Ag@ZIF-8 核壳异质结构纳米线对以上两种 类型的细菌均具有持续有效的抗菌活性.

“原位还原”法，即利用 MOFs 晶体结构存在的还原 性化学键来一步原位还原生成 M-NP, 无需添加表面活 性剂或其他还原性物质 ${ }^{[88,89]}$. 这种方法合成的金属纳米 粒子具有理化性质稳定、不易氧化、不团聚和反应活性 高等优点. 张继稳团队 ${ }^{[90]}$ 以环糊精 MOFs (CD-MOF)为 模板，合成了稳定的 AgNPs, 实现了高效的抗菌活性, 功能化修饰后能有效促进止血和伤口愈合(图 4c). 该研 究以生物相容性材料 CD-MOF 为模板, 利用结构中的 羟基抗衡离子 $(\mathrm{OH})$ 成功地合成了负载有超细(1.7 nm) AgNPs 的 CD-MOF (Ag@CD-MOF). 实验表明Ag@CDMOF 可在更低浓度时发挥抑菌作用, 其在 E. coli 和 $S$. aureus 中的最小抑菌浓度(MIC)分别为 16 和 $128 \mu \mathrm{g} / \mathrm{mL}$, 而市场上的 AgNPs 产品在 E. coli 和 S. aureus 中 MIC 为 $512 \mu \mathrm{g} / \mathrm{mL}$, 证明 $\mathrm{Ag} @ \mathrm{CD}-\mathrm{MOF}$ 的抗菌功效得到显著提 高. Ag@CD-MOF 微粒表面的环糊精单元经碳酸酯键化 学交联后, 将数量众多的超细 AgNPs 固定在微粒内, 实 现缓释. 研究人员采用 GS5 寡肽对交联的 CD-MOF 进 行表面修饰，促进血小板的止血功能，增强止血效果， 促进微粒在创伤部位的粘附, 协同 AgNPs 的抗菌作用, 促进伤口愈合.

硼(B)是一种宝贵的国家战略资源. B 含有一个空的 $\mathrm{p}$ 轨道, 使之成为较强的亲电物质和 Lewis 酸. 该特性 使其和细菌关键酶的活性位点形成配位键而产生亲和 力, 从而产生抗菌活性. 例如, 硼酸及其衍生物常作冷 湿敷用，具有消炎、抗菌、收敛及清洁创面作用. 2020 年, 宁桂玲团队 ${ }^{[64]}$ 通过在锌基硼咪唑框架材料(Zn-BIF) 结构中有意预留的活性位点, 成功制备了粒子粒径均 一、分布均匀的 $\mathrm{Ag}(\mathrm{Cu}) @ \mathrm{Zn}-\mathrm{BIF}$ ，同步实现了抗菌、硝 基苯酚化合物的快速催化还原和有机染料光催化降解 (图 4d). 该研究在室温条件下利用 Zn-BIF 结构中预留 B $-\mathrm{H}$ 键的还原能力, 无需任何还原剂的辅助, 原位合成 了包括 $\mathrm{Ag} 、 \mathrm{Pd} 、 \mathrm{Pt} 、 \mathrm{Au}$ 和 $\mathrm{Cu}$ 等在内的多种 M-NP, 合 成的 AgNPs 的尺寸约为 $9 \mathrm{~nm}$. 研究发现, Ag@Zn-BIF 和市售的 AgNPs 相比具有优异的抗菌性能, 对 E. coli 和 S. aureus 的 MIC 均在 30 50 $\mu \mathrm{g} / \mathrm{mL}$. Ag@Zn-BIF 抗 菌活性的产生除了 AgNPs 对细菌造成的机械损伤外, 实验中还发现 $\mathrm{Ag} @ \mathrm{Zn}$-BIF 可以生成大量的超氧自由基 $\left(\mathrm{O}^{2-}\right)$ 和 $\bullet \mathrm{OH}$, 在 AgNPs、嗍咪唑配体和 ROS 等多因素 协同作用下加速破坏细菌的结构, 从而实现高效抗菌. 除此以外, Ag@Zn-BIF 和 $\mathrm{Cu} @ \mathrm{Zn}-\mathrm{BIF}$ 分别在 2.5 和 6 min 内可将 4-硝基苯酚完全转化为 4-氨基苯酚. 这项工 作不仅开创了一种预留活性位点来原位合成多种贵金 属和非贵金属纳米粒子的方法, 合成的 M-NP@Zn-BIF 还可以用于处理多种生态环境问题. 
(a) Ship-in-the-bottle

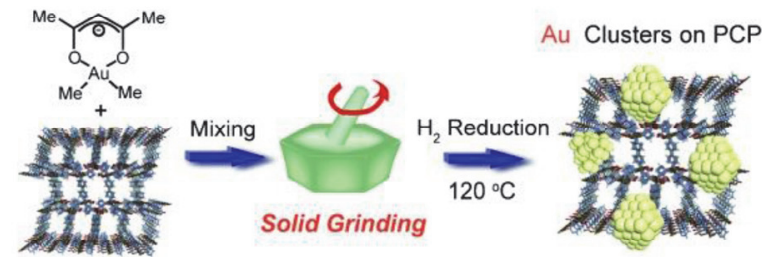

(c) In-situ reduction (b) Bottle-around-a-ship

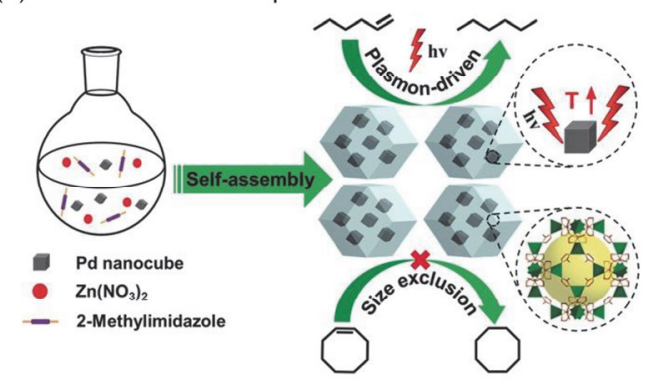

(d) In-situ reduction

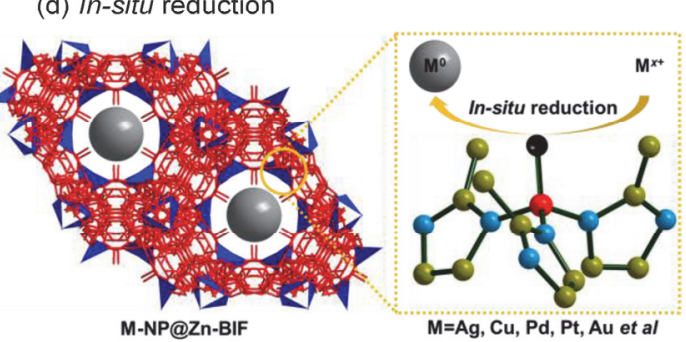

图 4 (a) 通过对 $\mathrm{Me}_{2} \mathrm{Au}(\mathrm{acac})$ 和 CPL-2 进行固体研磨和后续 $\mathrm{H}_{2}$ 还原合成 $\mathrm{Au} / \mathrm{CPL}-2$ 复合材料 ${ }^{[82]}$ (b) PdNCs@ZIF-8 的自组装和等离子体激元驱 动的选择性催化 ${ }^{[8]}$ 、 (c)超细 AgNPs 在 CD-MOF 的孔中生长和 CD-MOF 的表面改性的制备过程 ${ }^{[90]}$ 和(d) M-NP@Zn-BIF 的原位合成策略和 Zn-BIF 结构中的 $\mathrm{B}-\mathrm{H}$ 还原键 ${ }^{[64]}$

Figure 4 (a) Synthesis of Au/CPL-2 composite via solid grinding of CPL-2 with $\mathrm{Me}_{2} \mathrm{Au}(\mathrm{acac})$ followed by $\mathrm{H}_{2}$ reduction ${ }^{[82]}$ (Copyright 2008, Wiley-VCH), (b) self-assembly of Pd NCs@ZIF-8 and plasmon-driven selective catalysis ${ }^{[86]}$ (Copyright 2016, Wiley-VCH), (c) preparation process of ultrafine AgNPs growth in the pores of CD-MOF and the surface modification of CD-MOF ${ }^{[90]}$ (Copyright 2019, Wiley-VCH) and (d) in-situ synthesis strategy of M-NP@Zn-BIF and B-H reduction bond in Zn-BIF ${ }^{[64]}$ (Copyright 2020, Elsevier)

\section{5 药物缓释 MOFs 抗菌材料}

临床上抗菌药物的常规给药途径主要包括口腔、鼻 腔、皮肤、肺动脉或静脉注射. 抗菌药物为了作用于目 标组织, 一般要跨越多个生物屏障. 此外, 全身用药普 遍存在着药物溶解性差、稳定性有限、代谢快速、副作 用多以及生物利用度低等缺点, 治疗结果往往差强人 意. MOFs 的多孔结构可以有效地装载客体分子, 所以 目前的研究中通常将一些毒性较大或在体内容易被清 除的药物装载入 MOFs 的孔道中, 以达到避免频繁给药 及延长药物停留时间的目的. 浸润法是 MOFs 负载药物 最常见的方式之一. 浸润法是将 MOFs 浸润在药物溶液 中, 使得小分子药物包裹在其孔隙中, 再用溶剂去除多 余的游离药物分子. 这种方法要求小分子药物的尺寸与 MOFs 的孔径大致相符, 并且 MOFs 和药物分子之间的 亲和性较强 ${ }^{[91]}$. 与药物分子具有相互作用的特定金属 位点和有机官能团可以提高药物的运载效率. Serre 等 ${ }^{[2]}$ 利用浸润法将布洛芬成功地包裹到 MIL-100/101 孔 道中, 每个大孔和小孔的负载量分别为 92 和 56 个药物 分子, 实现了布洛芬的高效运载. 张强课题组 ${ }^{[93]}$ 利用 ZIF-8 设计出一种新型的载药系统来装载药物分子氟尿 嘧啶(5-Fu@ZIF), 其粒径约为 $200 \mathrm{~nm}$, 载药量高达 $21.1 \%$, 载药后纳米粒子比表面积由装载前的 $1570 \mathrm{~m}^{2} / \mathrm{g}$ 降低至装载后的 $110 \mathrm{~m}^{2} / \mathrm{g}$, 证明药物分子已成功填充至 孔道内部.

此外, MOFs 材料通常还可以进行外修饰, 这样不
但可以调节 MOFs 释放药物的行为, 还具有特异性靶向 功能, 避免药物分子过早断裂和降解, 促使更多药物有 效地渗透进细胞膜内, 以增加病变处的药物的有效释放 量, 从而提高治疗效果. MOFs 作为药物载体的优点还 在于可通过改变有机配体的种类而赋予材料不同的性 质, 以达到响应释放药物的目的, 从而进一步避免药物 分子在穿越生物屏障过程中的提前释放. 例如, 伤口细 菌感染处或肿瘤生长处往往 $\mathrm{H}_{2} \mathrm{O}_{2}$ 浓度偏高, 而酸性也 偏强, 可以利用这种特定的生理环境来提高分子在目标 位置的靶向蓄积.

Van 是一种糖肽类抗生素, 是用来治疗多种细菌感 染的抗细菌药物 ${ }^{[94]}$. 治疗皮肤感染、败血症、心内膜炎、 骨关节感染以及因 MRSA 引起的脑膜炎时, 静脉注射 通常作为一线治疗方案而使用, 给药时的使用剂量将依 患者自身情况做调整. Van 也是一种治疗严重伪膜性结 肠炎的口服药, 然而口服时药效将会大为降低. MOFs 已经用于传递 Van, 这是因为 MOFs 具有丰富的基团作 为活性位点. 通过改性, 对缓释进行调节以达到最佳的 治疗效果. 吴水林团队 ${ }^{[95]}$ 报道了一种具有持久抗菌治 疗功能且无毒的 MOF-53(Fe)@Van 复合材料. 实验结果 表明, MOF-53(Fe)@Van 具有可控的缓释药物性能, 对 S. aureus 具有较好的抗菌效率(杀菌率大于 $90 \%$ )、良好 的化学稳定性和近乎可忽略的细胞毒性. 有趣的是, 在 MOF-53(Fe) 降解过程中 $\mathrm{Fe}^{3+}$ 的持续释放促进了 MC3T3 细胞的增殖和成骨分化. 此后, 王佰亮课题组 ${ }^{[96]}$ 于 2019 年报道了一种 $\mathrm{pH}$ 响应型 ZIF-8-PAA-MB@AgNPs@Van- 
PEG, 用于治疗眼内感染(图 5a). 含有聚丙烯酸的 ZIF-8 核层确保了广谱抗菌剂甲基苯蓝( $\mathrm{MB}$ )的高效负载和 $\mathrm{pH}$ 响应释放, 负载 $\mathrm{MB}$ 后, 通过 $\mathrm{AgNO}_{3}$ 和多巴胺对载体进 行修饰, 原位形成 AgNPs 以增强抗菌性能, 然后用 $\mathrm{Van} / \mathrm{NH}_{2}-\mathrm{PEG}$ 进行二次修饰, 进一步增强纳米粒子的抗 感染活性. 体外实验表明, 复合材料在 $650 \mathrm{~nm}$ 激光照射 下对 E. coli, S. aureus 和 MRSA 具有高效灭活作用且能 够完全清除生物被膜. 同时, 体外视网膜色素上皮细胞 生物相容性实验和治疗眼内感染小鼠模型实验进一步 证实了 MOFs 复合体系具有优异的生物相容性和高效的 抗菌抗感染功能.

除了 Van 等抗生素之外, MOFs 还常常被用来递送 $\beta$-内酰胺酶抑制剂、3-叠氮基- $d$-丙氨酸和头孢他啶等具 有抗菌功能的药物试剂. 舒巴坦(Sulbactam, Sul)作为一 种不可逆的竞争性 $\beta$-内酰胺酶抑制剂, 对革兰阳性及阴

(a) Vancomycin

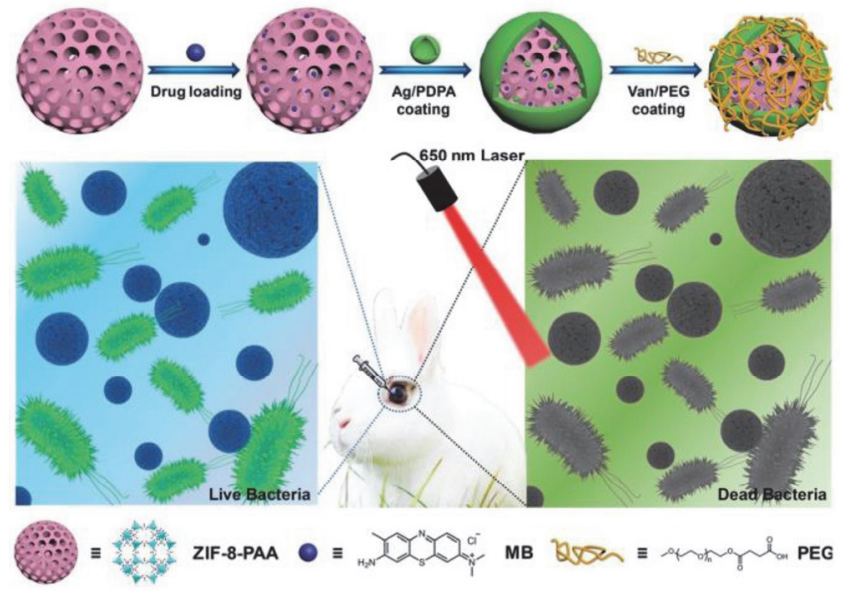

(c) D-AzAla

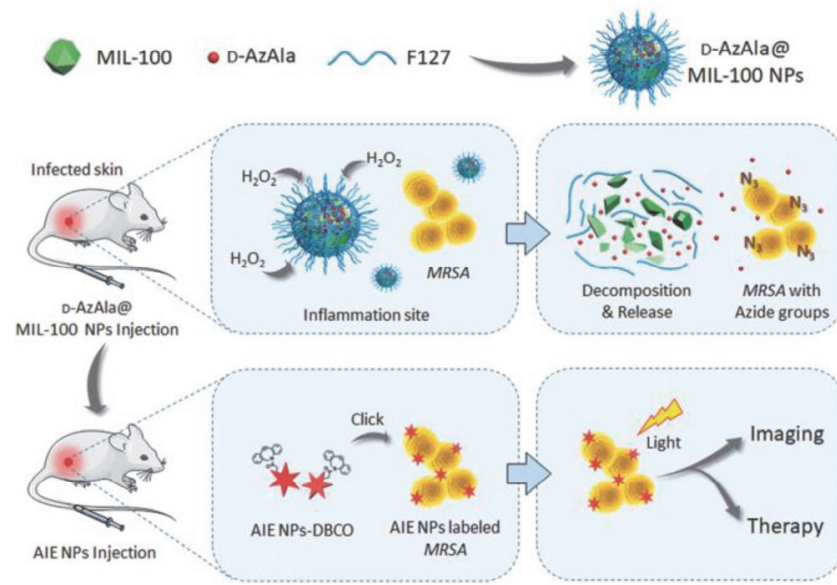

性菌(除绿脓杆菌外)所产生的 $\beta$-内酰胺酶均有抑制作 用，通常与青霉素类及头孢菌素类药物合用. Sul 与氨苄 西林可用于联合治疗敏感细菌所致的关节感染以及败 血症和脑膜炎等疾病. 在临床使用过程中, Sul 的全身性 释放一直是一个亟待解决的关键问题. 张金超课题 组 ${ }^{[97]}$ 为了解决这一问题, 设计了一种负载 Sul 的 MOFs 纳米粒子以治疗 MRSA 引起的全身感染(图 5b). 他们首 先将 Sul 载于以 $\mathrm{Fe}^{3+}$ 和羧茮西林形成的 MOFs 孔道中. 外层的 MOFs 可以有效地保护内部的 Sul, 从而避免了 Sul 的提前释放. 实验结果表明, MOFs 纳米粒子在 MRSA 感染造成的酸性条件下 $(\mathrm{pH}=5.0)$ 可以有效地抑 制并杀灭 MRSA 及其形成的生物被膜. 此外，体内实验 也证明了通过静脉注射 MOFs 纳米粒子, 能有效治疗 MRSA 引起的皮肤感染和全身性感染小鼠，并提高了全 身性感染小鼠的生存率.

(b) Sulbactam

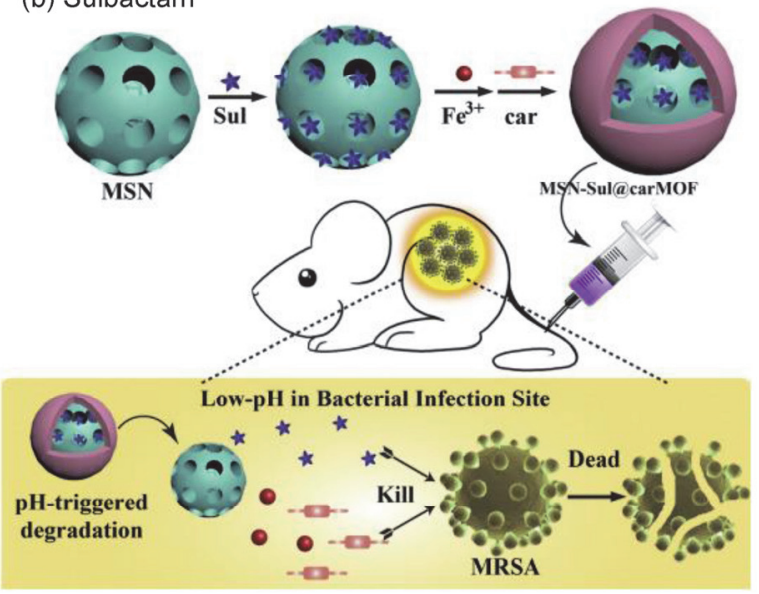

(d) Peroxidase
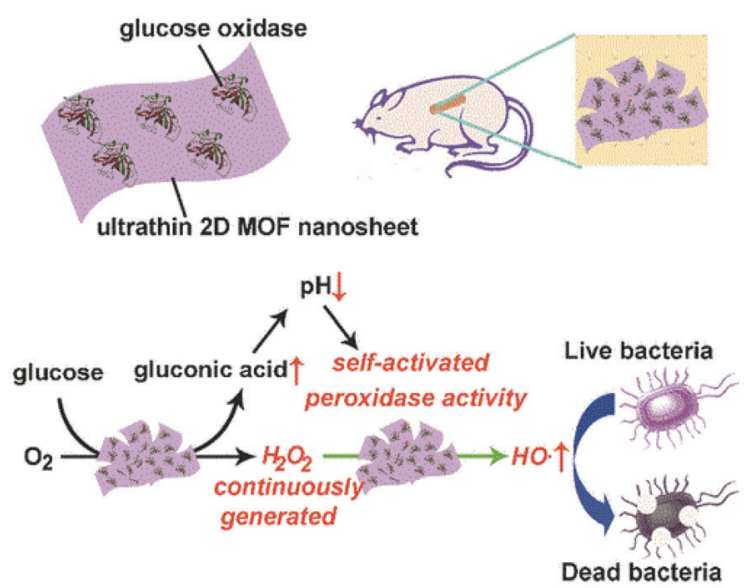

图 5 (a) ZIF-8-PAA-MB@AgNPs@Van-PEG 的合成及其在眼内感染治疗中的应用 ${ }^{[96]}$ 、(b)在较低 $\mathrm{pH}$ 值条件下, $\mathrm{Fe}^{3+} \mathrm{MOF}$ 涂层的设计及在细菌感 染位置同步释放抗生素和抑制剂机理示意图 ${ }^{[97]}$ 、(c) MIL-100(Fe)纳米粒子的制备及用于影像引导下的体内精确抗菌疗法策略示意图 ${ }^{[98]}$ 和(d)二维 $\mathrm{MOF} / \mathrm{GO}_{x}$ 纳米催化剂的设计及抗菌机理示意图 ${ }^{[104]}$

Figure 5 (a) The synthesis of ZIF-8-PAA-MB@AgNPs@Van-PEG and the application of endophthalmitis treatment ${ }^{[96]}$ (Copyright 2019, Wiley-VCH), (b) schematic of the design of car/ $/ \mathrm{Fe}^{3+} \mathrm{MOF}$-coated MSN and the synchronous release of antibiotic molecules and inhibitors at the bacterial infection site at low $\mathrm{pH}^{[97]}$ (Copyright 2017, Elsevier), (c) the fabrication of the nanoplatform based on MIL-100(Fe) and the proposed strategy for image-guided precise

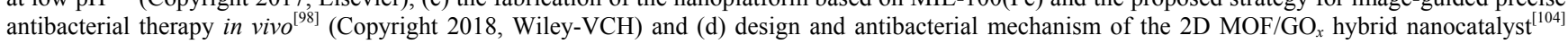
(Copyright 2019, American Chemical Society) 
2018 年, 刘斌团队 ${ }^{[98]}$ 设计了一种负载有 3-叠氮基$d$-丙氨酸( $D$-AzAla) 的 MOFs 纳米粒子 $[D-A z A l a @ M I L-$ $100(\mathrm{Fe})$ ], 并通过具有聚集诱导发光(AIE)性质的分子来 实现细菌体内代谢荧光标记引导下的精准抗菌治疗 (图 5 c). 使用 $\mathrm{Fe}^{3+}$ 和均苯三酸 (TMA)构建了直径约为 120 $\mathrm{nm}$ 的 MIL-100(Fe)纳米粒子并负载 $D$-AzAla, 经静脉注 射后, MOFs 可通过高渗透长滞留效应(Enhanced Permeability and Retention Effect, EPR) 在 $\mathrm{H}_{2} \mathrm{O}_{2}$ 高表达的炎 症区域积聚, 此时 $\mathrm{Fe}^{3+}$ 和 TMA 之间的配位键被 $\mathrm{H}_{2} \mathrm{O}_{2}$ 破坏, 从而导致 MIL-100(Fe)的体内降解和 D-AzAla 的 释放. 同时, $D$-AzAla 与细菌细胞壁结合并暴露出叠氮 化物基团. 具有 AIE 性质的分子 2-(1-(5-(4-(1,2,2-三(4甲氧基苯基)乙烯基)苯基)噻吩-2-基)亚乙基)丙二腈可 与裸露在外的叠氮化物基团结合, 在体内同时实现细菌 的荧光标记和高效的抗菌活性. 这项工作首次将非天然 官能团与体内细菌特异性地结合在一起, 此类探针分子 为细菌感染的可视化治疗和诊疗一体化提供了一种新 的策略.

随后, Sava Gallis 等 ${ }^{[99]}$ 报道了另一个药物缓释治疗 平台. 他们将抗菌药物头孢他啶封装到 ZIF-8 中以治疗 细胞内感染. 头孢他啶对多种耐药革兰阴性杆菌引起的 免疫缺陷者感染、医院内感染以及革兰阴性杆菌或 $P$. aeruginosa 所致中枢神经系统感染尤为适用. 实验结果 表明, 头孢他啶的释放并非依赖于 ZIF-8 在细胞内的生 物降解, 并可以实现长达一周的药物释放. 头孢他啶 @ CZIF-8 对巨噬 A549 细胞和肺上皮 RAW 264.7 细胞系 表现出良好的生物相容性, 并且对 E. coli 具有出色的抗 菌活性. 更重要的是, 由于 ZIF-8 中有机配体咪唑的发 光性能, 使用共聚焦显微镜 $\mathrm{z}$-stacks 进行 3D 重建, 证实 了头孢他啶@ZIF-8 在细胞中的内化作用, 并监测了药 物缓释过程. 该项工作利用 ZIF-8 固有的自发光性能来 监测药物缓释, 而不是引入其他染色剂, 避免了材料设 计体系的复杂性以及染料泄露所带来的弊端.

除了药物以外, 天然酶也能够利用其独特的化学活 性对细菌造成不可逆转的损伤. 不过, 天然酶活性不稳 定及提纯成本较高等缺点限制了其实际抗菌应用和成 果转化. 当前, 科研工作者们正致力于研发能够仿生天 然酶的性能甚至超越天然酶活性的新型纳米酶材料. 这 种具有类自然酶活性的纳米材料为抗菌和抗生物被膜 带来了崭新的研究思路 ${ }^{[100]}$. 具有类氧化还原酶活性的 纳米酶通过诱导产生高活性的 ROS 来有效杀灭细 菌 ${ }^{[101,102]}$. 纳米酶能够调节体内 $\cdot \mathrm{OH}$ 或 $\mathrm{O}^{2-}$ 的水平. 一方 面, 具有类过氧化物酶活性的纳米酶催化低浓度的 $\mathrm{H}_{2} \mathrm{O}_{2}$ 产生・ OH. 另一方面, 具有类有氧化物酶活性的纳 米酶催化 $\mathrm{O}_{2}$ 生成 $\mathrm{O}^{2-}$. 利用这些种类 ROS 所具有的强 氧化性能, 破坏细菌以及生物膜中的重要生物分子, 从 而实现高效的杀菌活性和抗生物膜效果 ${ }^{[103]}$. 而且, 这 种抗菌方式也不会导致细菌耐药性的产生.

基于 MOFs 装载过氧化物纳米酶的应用较少, 其中
一个主要原因是纳米酶催化的最佳 $\mathrm{pH}$ 值为 $3 \sim 4$ 的强酸 性环境，这严重限制了纳米酶在人体体液平衡条件下的 应用. 此外, 若直接引入高浓度的 $\mathrm{H}_{2} \mathrm{O}_{2}$ 也会对正常组织 造成意想不到的损伤. 2019 年, 曲晓刚团队 ${ }^{[104]}$ 选用超薄 的 $\mathrm{MOFs}$ 纳米片 $[2 \mathrm{D} \mathrm{CuTCPP}(\mathrm{Fe})]$ 负载葡萄糖氧化酶 $\left(\mathrm{GO}_{x}\right)$ 制备了这种混合型纳米催化剂(图 5d). 其中, $\mathrm{GO}_{x}$ 会使得葡萄糖转化为葡萄糖酸和 $\mathrm{H}_{2} \mathrm{O}_{2}$, 提高了感染处 $\mathrm{H}_{2} \mathrm{O}_{2}$ 的浓度, 而且生成的葡萄糖酸将 $\mathrm{pH}$ 值从 7 降低到 3 4, 进而激活 $2 \mathrm{D} \mathrm{Cu}-\mathrm{TCPP}(\mathrm{Fe})$ 纳米片的过氧化物纳 米酶的生物活性. 生成的 $\mathrm{H}_{2} \mathrm{O}_{2}$ 也能用于激活 $2 \mathrm{D}$ $\mathrm{Cu}-\mathrm{TCPP}(\mathrm{Fe})$ 纳米片进行后续的催化反应, 高效地生成 • $\mathrm{OH}$, 进而实现体内高效抗菌.

\section{6 总结与展望}

随着现代合成技术的快速发展以及多学科间的密 切交叉, MOFs 基功能材料得到了快速的发展，特别是 近年来在药物缓释与代谢、生命体活动实时监测和恶性 肿瘤或耐药菌感染等重大疾病的诊断与治疗等方面受 到广泛关注. 近年来, 基于 MOFs 的相关抗菌研究取得 了阶段性成果. 这篇综述详细地介绍了 MOFs 抗菌材料 的最新进展, 从细菌耐药性产生机理出发, 分类概述了 包括不同金属中心和配体 MOFs 材料、MOFs 包覆金属 纳米粒子材料和药物缓释 MOFs 材料等在抗菌、伤口促 愈等领域中的一些应用.

尽管MOFs 抗菌材料在实验室研究中已经取得了部 分重要突破, 但 MOFs 并没有完全发挥其在结构和性能 上的优势. MOFs 在抗菌抗感染研究领域中仍然有以下 几方面的局限性亟待解决: (1) MOFs 作为基于金属-配 位作用构建的有序晶态框架材料, 因降解而产生的金属 离子和有机配体对生物体的毒理作用需要进行审慎的 评估，这是在投入临床实验之前最迫切需要阐明的问 题. 使用具有生物活性的有机分子作为配体和人体必需 的微量元素作为金属节点来构筑单元结构, 对避免 MOFs 生物毒性具有重要的指导意义. 此外, 对 MOFs 的表面进行功能化修饰以调节表面电势和结合亲和力 也是解决此类问题的方法之一. (2)如何按需调控 MOFs 在生物体内的稳定性也需要研究. 配体与金属之间形成 稳定的配位键是 MOFs 结构形成的一个关键作用力, 而 且配体与配体之间也存在分子间相互作用力 (如氢键等 原子间较弱的相互作用). MOFs 在复杂的生理条件下容 易分解, 这既是其优点, 也是不可忽视的缺点, 因此根 据实际需求来调控 MOFs 的稳定性非常重要. (3)如何实 现药物的靶向递送与精准释放也是实际应用面临的一 大挑战. 目前大多数 MOFs 体系在药物递送过程中会有 一定程度的药物泄露, 导致药效降低. 此外, 药物释放 的动力学规律还不是十分清楚. 通过合理的表面修饰 (如核酸适配体、透明质酸等主动靶向制剂)，有望降低 活性药物泄露, 同时还能赋予纳米粒子主动靶向能力. 
(4) MOFs 小批量合成限制了其大规模应用, 特别是制 备条件苛刻、流程复杂的 MOFs 复合材料, 如何使得 MOFs 合成制备轻量化, 提高其大规模医学临床应用的 潜力还需深入研究. 此外, 对于 MOFs 基抗菌喷剂、抗 菌纤维膜的研究还比较少, 也制约着其实际应用.

综上所述, MOFs 具有许多传统多孔材料不具备的 优良理化性质, 无论是在抗肿瘤还是在抗菌抗感染等重 大疾病诊疗领域都有着相当广泛的应用前景. 尽管当前 还有一些尚未解决的问题, 但是随着 MOFs 的研究越来 越深入，这些困难和挑战一定会被逐一攻克.

\section{作者简介}

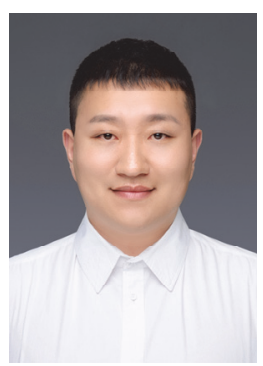

齐野, 博士研究生, 2017 年毕业于大连海事大学, 获理学 硕士学位. 2017 年起于大连理工大学化工学院攻读博士学位. 主要研究方向为含㸴金属有机框架复合材料的结构构筑及其 在抗菌、抗癌等生物领域上的应用.

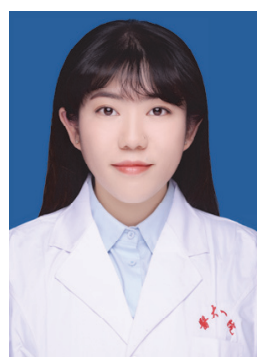

任双颂, 2017 年毕业于英国邓迪大学, 获理学硕士学位. 2018 年起就职于大连医科大学附属第一医院. 目前主要研究 方向为多模态成像引导下恶性肿瘤的微创热消融研究.

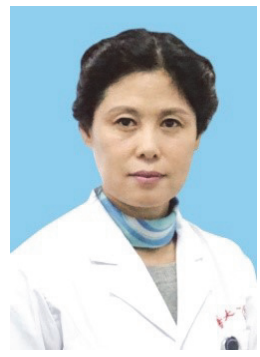

车颖, 教授, 博士生导师, 大连医科大学附属第一医院超 声科主任. 中国超声医学工程学会委员、超声介入委员会委员 及中国抗癌协会肿瘤微创治疗专业委员会委员。目前主要从 事良、恶性肿瘤的微创热消融治疗.

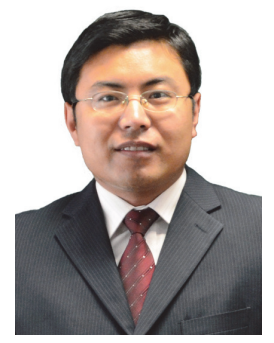

叶俊伟, 教授, 博士生导师, 大连理工大学化工学院副院 长. 2007 年毕业于吉林大学, 获博士学位. 发表学术论文 100 余篇, 主要从事资源化工和先进功能材料研究.

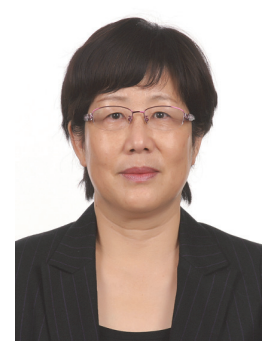

宁桂玲, 教授, 博士生导师, 辽宁省硼镁资源化工与新材 料工程中心主任. 1996 年毕业于大连理工大学, 获博士学位. 在 Angew. Chem., Int. Ed., J. Am. Chem. Soc. 和 Chem. Commun. 等国际化学期刊发表学术论文 200 余篇. 研究领域主要包括 资源化工、能源和生物功能材料.

\section{References}

[1] Tan, L.; Li, J.; Liu, X.; Cui, Z.; Yang, X.; Yeung, K. W. K.; Pan, H.; Zheng, Y.; Wang, X.; Wu, S. Small 2018, 14, 1703197.

[2] Rtimi, S.; Dionysiou, D. D.; Pillai, S. C.; Kiwi, J. Appl. Catal., B 2019, 240, 291.

[3] Alseth, E. O.; Pursey, E.; Lujan, A. M.; McLeod, I.; Rollie, C.; Westra, E. R. Nature 2019, 574, 549.

[4] Tang, S.; Zheng, J. Adv. Healthcare Mater. 2018, 7, 1701503.

[5] Qi, Y.; Ye, J.; Zhang, S.; Tian, Q.; Xu, N.; Tian, P.; Ning, G. J. Alloys Compd. 2019, 782, 780 .

[6] Chai, Z.; Tian, Q.; Ye, J.; Zhang, S.; Wang, G.; Qi, Y.; Che, Y.; Ning, G. J. Mater. Sci. 2020, 55, 4408 .

[7] Ye, J.; Cheng, H.; Li, H.; Yang, Y.; Zhang, S.; Rauf, A.; Zhao, Q.; Ning, G. J. Colloid Interface Sci. 2017, 504, 448.

[8] Peng, K.; Ding, W.; Tu, W.; Hu, J.; Liu, C.; Yang, J. Acta Chim. Sinica 2016, 74, 713. (彭开美，丁伟，涂伟萍，胡剑青，Liu Chao, Yang Jian, 化学学报, 2016, 74, 713.)

[9] Hook, A. L.; Chang, C.-Y.; Yang, J.; Atkinson, S.; Langer, R.; Anderson, D. G.; Davies, M. C.; Williams, P.; Alexander, M. R. Adv. Mater. 2013, 25, 2542.

[10] Wang, K.; He, J. Acta Chim. Sinica 2018, 76, 807. (王凯凯, 贺军 辉, 化学学报, 2018, 76, 807.)

[11] Furukawa, H.; Cordova, K. E.; O'Keeffe, M.; Yaghi, O. M. Science 2013, 341, 974

[12] Rowsell, J. L. C.; Yaghi, O. M. Angew. Chem., Int. Ed. 2005, 44, 4670 .

[13] Zhang, X.; Wang, X.; Fan, W.; Sun, D. Chin. J. Chem. 2020, 38, 509.

[14] Wang, X.; Zhang, Y.; Chang, Z.; Huang, H.; Liu, X.-T.; Xu, J.; Bu, X.-H. Chin. J. Chem. 2019, 37, 871 .

[15] Schoedel, A.; Li, M.; Li, D.; O'Keeffe, M.; Yaghi, O. M. Chem. Rev. 2016, 116, 12466.

[16] Yaghi, O. M.; Li, H. L.; Davis, C.; Richardson, D.; Groy, T. L. Acc. Chem. Res. 1998, 31, 474

[17] Zeng, J.; Wang, X.; Zhang, X.; Zhuo, R. Acta Chim. Sinica 2019, 77, 1156. (曾锦跃, 王小双, 张先正, 卓仁禧, 化学学报, 2019, 77, 1156.) 
[18] Cao, L.; Wang, T.; Wang, C. Chin. J. Chem. 2018, 36, 754.

[19] Gao, B.; Zhou, J.; Wang, H.; Zhang, G.; He, J.; Xu, Q.; Li, N.; Chen, D.; Li, H.; Lu, J. Chin. J. Chem. 2019, 37, 148.

[20] Guo, X.; Chen, X.; Su, D.; Liang, C. Acta Chim. Sinica 2018, 76, 22. (郭小玲, 陈霄, 苏党生, 梁长海, 化学学报, 2018, 76, 22.)

[21] Wu, Z.; Shi, Y.; Li, C.; Niu, D.; Chu, Q.; Xiong, W.; Li, X. Acta Chim. Sinica 2019, 77, 758. (武卓敏, 石勇, 李春艳, 牛丹阳, 楚 奇, 熊巍, 李新勇, 化学学报, 2019, 77, 758.)

[22] Luo, Y.; Li, J.; Liu, X.; Tan, L.; Cui, Z.; Feng, X.; Yang, X.; Liang, Y.; Li, Z.; Zhu, S.; Zheng, Y.; Yeung, K. W. K.; Yang, C.; Wang, X.; Wu, S. ACS Cent. Sci. 2019, 5, 1591.

[23] Yang, Y.; Deng, Y.; Huang, J.; Fan, X.; Cheng, C.; Nie, C.; Ma, L.; Zhao, W.; Zhao, C. Adv. Funct. Mater. 2019, 29, 1900143.

[24] Yao, X.; Zhu, G.; Zhu, P.; Ma, J.; Chen, W.; Liu, Z.; Kong, T. Adv. Funct. Mater. 2020, 30, 1909389.

[25] Nasrabadi, M.; Ghasemzadeh, M. A.; Monfared, M. R. Z. New J. Chem. 2019, 43, 16033.

[26] Chen, M.; Long, Z.; Dong, R.; Wang, L.; Zhang, J.; Li, S.; Zhao, X.; Hou, X.; Shao, H.; Jiang, X. Small 2020, 16, 1906240.

[27] Alexander, F. Br. J. Exp. Pathol. 1929, 10, 226.

[28] Lacombe, S.; Rougon-Cardoso, A.; Sherwood, E.; Peeters, N.; Dahlbeck, D.; van Esse, H. P.; Smoker, M.; Rallapalli, G.; Thomma, B. P. H. J.; Staskawicz, B.; Jones, J. D. G.; Zipfel, C. Nat. Biotechnol. 2010, 28, 365 .

[29] Jiao, Y.; Zhang, X. Acta Chim. Sinica 2018, 76, 659. (焦阳, 张希, 化学学报, 2018, 76, 659.)

[30] Zhang, Q.-Q.; Ying, G.-G.; Pan, C.-G.; Liu, Y.-S.; Zhao, J.-L. Environ. Sci. Technol. 2015, 49, 6772

[31] Molton, J. S.; Tambyah, P. A.; Ang, B. S. P.; Ling, M. L.; Fisher, D. A. Clin. Infect. Dis. 2013, 56, 1310.

[32] Magiorakos, A. P.; Srinivasan, A.; Carey, R. B.; Carmeli, Y.; Falagas, M. E.; Giske, C. G.; Harbarth, S.; Hindler, J. F.; Kahlmeter, G.; Olsson-Liljequist, B.; Paterson, D. L.; Rice, L. B.; Stelling, J.; Struelens, M. J.; Vatopoulos, A.; Weber, J. T.; Monnet, D. L. Clin. Microbiol. Infect. 2012, 18, 268.

[33] Luria, S. E.; Delbrück, M. Genetics 1943, 28, 491.

[34] Long, H.; Miller, S. F.; Strauss, C.; Zhao, C.; Cheng, L.; Ye, Z.; Griffin, K.; Te, R.; Lee, H.; Chen, C.-C.; Lynch, M. PNAS 2016, $113, \mathrm{E} 2498$.

[35] Gutierrez, A.; Laureti, L.; Crussard, S.; Abida, H.; Rodriguez-Rojas, A.; Blazquez, J.; Baharoglu, Z.; Mazel, D.; Darfeuille, F.; Vogel, J.; Matic, I. Nat. Commun. 2013, 4, 1610.

[36] Bjedov, I.; Tenaillon, O.; Gerard, B.; Souza, V.; Denamur, E.; Radman, M.; Taddei, F.; Matic, I. Science 2003, 300, 1404.

[37] Yun, B.-R.; Malik, A.; Kim, S. B. Gene 2020, 733, 144379.

[38] Tabashnik, B. E.; Huang, F.; Ghimire, M. N.; Leonard, B. R.; Siegfried, B. D.; Rangasamy, M.; Yang, Y.; Wu, Y.; Gahan, L. J.; Heckel, D. G.; Bravo, A.; Soberon, M. Nat. Biotechnol. 2011, 29, 1128.

[39] Dey, B.; Dey, R. J.; Cheung, L. S.; Pokkali, S.; Guo, H.; Lee, J.-H.; Bishai, W. R. Nat. Med. 2015, 21, 401.

[40] Thaker, M. N.; Wang, W.; Spanogiannopoulos, P.; Waglechner, N.; King, A. M.; Medina, R.; Wright, G. D. Nat. Biotechnol. 2013, 31, 922.

[41] Dodd, M. C.; Kohler, H.-P. E.; Von Gunten, U. Environ. Sci. Technol. 2009, 43, 2498.

[42] Kim, J.; Pitts, B.; Stewart, P. S.; Camper, A.; Yoon, J. Antimicrob. Agents Chemother. 2008, 52, 1446.

[43] Yan, D.; Wu, X.; Pei, J.; Wu, C.; Wang, X.; Zhao, H. Ceram. Int. 2020, 46, 696

[44] Hu, X. N.; Zhao, Y. Y.; Hu, Z. J.; Saran, A.; Hou, S.; Wen, T.; Liu, W. Q.; Ji, Y. L.; Jiang, X. Y.; Wu, X. C. Nano Res. 2013, 6, 822.

[45] Zhu, M.; Li, X.; Ge, L.; Zi, Y.; Qi, M.; Li, Y.; Li, D.; Mu, C. Mater. Sci. Eng., C 2020, 106, 110185.

[46] Berchel, M.; Gall, T. L.; Denis, C.; Hir, S. L.; Quentel, F.; Elléouet, C.; Montier, T.; Rueff, J.-M.; Salaün, J.-Y.; Haelters, J.-P.; Hix, G. B.; Lehn, P.; Jaffrès, P.-A. New J. Chem. 2011, 35, 1000

[47] Lu, X. Y.; Ye, J. W.; Sun, Y.; Bogale, R. F.; Zhao, L. M.; Tian, P.; Ning, G. L. Dalton Trans. 2014, 43, 10104.

[48] Lu, X. Y.; Ye, J. W.; Zhao, L. M.; Lin, Y.; Ning, G. L. J. Coord. Chem. 2014, 67, 1133.

[49] Rauf, A.; Ye, J. W.; Hao, A. Y.; Zhao, L. Y.; Zhang, S. Q.; Qi, Y.; Shi, L.; Ning, G. L. J. Coord. Chem. 2018, 71, 3266.

[50] Zhang, S.; Ye, J.; Sun, Y.; Kang, J.; Liu, J.; Wang, Y.; Li, Y.; Zhang, L.; Ning, G. Chem. Eng. J. 2020, 390, 124523.

[51] Panchal, P.; Paul, D. R.; Sharma, A.; Choudhary, P.; Meena, P.; Nehra, S. P. J. Colloid Interface Sci. 2020, 563, 370.
[52] Abendrot, M.; Checinska, L.; Kusz, J.; Lisowska, K.; Zawadzka, K.; Felczak, A.; Kalinowska-Lis, U. Molecules 2020, 25, 951.

[53] Li, P.; Li, J.; Feng, X.; Li, J.; Hao, Y.; Zhang, J.; Wang, H.; Yin, A.; Zhou, J.; Ma, X.; Wang, B. Nat. Commun. 2019, 10, 2177.

[54] Mallick, S.; Sharma, S.; Banerjee, M.; Ghosh, S. S.; Chattopadhyay, A.; Paul, A. ACS Appl. Mater. Interfaces 2012, 4, 1313.

[55] Chen, S.; Tang, F.; Tang, L.; Li, L. ACS Appl. Mater. Interfaces 2017, 9, 20895.

[56] Rauf, A.; Ye, J. W.; Zhang, S. Q.; Shi, L.; Akram, M. A.; Ning, G. L. Polyhedron 2019, 166, 130.

[57] Han, D.; Han, Y.; Li, J.; Liu, X.; Yeung, K. W. K.; Zheng, Y.; Cui, Z.; Yang, X.; Liang, Y.; Li, Z.; Zhu, S.; Yuan, X.; Feng, X.; Yang, C.; Wu, S. Appl. Catal., B 2020, 261, 118248.

[58] Lu, X.; Ye, J.; Zhang, D.; Xie, R.; Bogale, R. F.; Sun, Y.; Zhao, L.; Zhao, Q.; Ning, G. J. Inorg. Biochem. 2014, 138, 114.

[59] Liu, Y.; Xu, X.; Xia, Q.; Yuan, G.; He, Q.; Cui, Y. Chem. Commun. 2010, 46, 2608.

[60] Kirillov, A. M.; Wieczorek, S. W.; Lis, A.; Guedes da Silva, M. F. C.; Florek, M.; Król, J.; Staroniewicz, Z.; Smoleński, P.; Pombeiro, A. J. L. Cryst. Growth Des. 2011, 11, 2711.

[61] Akbarzadeh, F.; Motaghi, M.; Chauhan, N. P. S.; Sargazi, G. Heliyon 2020, 6, e03231.

[62] Ahmad, N.; Samavati, A.; Nordin, N. A. H. M.; Jaafar, J.; Ismail, A. F.; Malek, N. A. N. N. Sep. Purif. Technol. 2020, 239, 116554.

[63] Yang, Y.; Guo, Z.; Huang, W.; Zhang, S.; Huang, J.; Yang, H.; Zhou, Y.; Xu, W.; Gu, S. Appl. Surf. Sci. 2020, 503, 144079.

[64] Qi, Y.; Ye, J.; Ren, S.; Lv, J.; Zhang, S.; Che, Y.; Ning, G. J. Hazard. Mater. 2020, 387, 121687.

[65] Abednejad, A.; Ghaee, A.; Nourmohammadi, J.; Mehrizi, A. A. Carbohydr. Polym. 2019, 222, 115033.

[66] Majumdar, D.; Das, D.; Sreejith, S. S.; Das, S.; Kumar Biswas, J.; Mondal, M.; Ghosh, D.; Bankura, K.; Mishra, D. Inorg. Chim. Acta 2019, 489, 244.

[67] Azad, F. N.; Ghaedi, M.; Dashtian, K.; Hajati, S.; Pezeshkpour, V. Ultrason. Sonochem. 2016, 31, 383.

[68] Abbasi, A. R.; Akhbari, K.; Morsali, A. Ultrason. Sonochem. 2012, $19,846$.

[69] Zhang, Q.; Yue, C.; Zhang, Y.; Lü, Y.; Hao, Y.; Miao, Y.; Li, J.; Liu, Z. Inorg. Chim. Acta 2018, 473, 112.

[70] Usefi, S.; Akhbari, K.; White, J. J. Solid State Chem. 2019, $276,61$.

[71] Abbasloo, F.; Khosravani, S. A.; Ghaedi, M.; Dashtian, K.; Hosseini, E.; Manzouri, L.; Khorramrooz, S. S.; Sharifi, A.; Jannesar, R.; Sadri, F. Ultrason. Sonochem. 2018, 42, 237.

[72] Shi, Z.; Zhang, K.; Zada, S.; Zhang, C.; Meng, X.; Yang, Z.; Dong, H. ACS Appl. Mater. Interfaces 2020, 12, 12600.

[73] Ni, K.; Luo, T.; Lan, G.; Culbert, A.; Song, Y.; Wu, T.; Jiang, X.; Lin, W. Angew. Chem., Int. Ed. 2020, 59, 1108.

[74] Zheng, X.; Wang, L.; Guan, Y.; Pei, Q.; Jiang, J.; Xie, Z. Biomaterials 2020, 235, 119792.

[75] Liu, M.; Wang, L.; Zheng, X.; Xie, Z. ACS Appl. Mater. Interfaces 2017, 9, 41512.

[76] Engell, R. E.; Lim, S. S. Lancet 2013, 381, S44.

[77] Fabrega, J.; Luoma, S. N.; Tyler, C. R.; Galloway, T. S.; Lead, J. R. Environ. Int. 2011, 37, 517.

[78] Yan, Z.; Fu, L.; Zuo, X.; Yang, H. Appl. Catal., B 2018, 226, 23.

[79] Park, C. M.; Chu, K. H.; Heo, J.; Her, N.; Jang, M.; Son, A.; Yoon, Y. J. Hazard. Mater. 2016, 309, 133.

[80] Bagheri, N.; Khataee, A.; Hassanzadeh, J.; Habibi, B. J. Hazard. Mater. 2018, 360, 233.

[81] Howarth, A. J.; Liu, Y.; Li, P.; Li, Z.; Wang, T. C.; Hupp, J.; Farha, O. K. Nat. Rev. Mater. 2016, 1, 15018.

[82] Ishida, T.; Nagaoka, M.; Akita, T.; Haruta, M. Chem.-Eur. J. 2008, 14,8456 .

[83] Duan, C.; Liu, C.; Meng, X.; Gao, K.; Lu, W.; Zhang, Y.; Dai, L.; Zhao, W.; Xiong, C.; Wang, W.; Liu, Y.; Ni, Y. Carbohydr. Polym. 2020, 230, 115642.

[84] Whitford, C. L.; Stephenson, C. J.; Gomez-Gualdron, D. A.; Hupp, J. T.; Farha, O. K.; Snurr, R. Q.; Stair, P. C. J. Phys. Chem. C 2017, $121,25079$.

[85] Mukoyoshi, M.; Kobayashi, H.; Kusada, K.; Hayashi, M.; Yamada, T.; Maesato, M.; Taylor, J. M.; Kubota, Y.; Kato, K.; Takata, M.; Yamamoto, T.; Matsumura, S.; Kitagawa, H. Chem. Commun. 2015, $51,12463$.

[86] Yang, Q.; Xu, Q.; Yu, S.-H.; Jiang, H.-L. Angew. Chem., Int. Ed. 2016, 55, 3685 .

[87] Guo, Y.-F.; Fang, W.-J.; Fu, J.-R.; Wu, Y.; Zheng, J.; Gao, G.-Q.; 
Chen, C.; Yan, R.-W.; Huang, S.-G.; Wang, C.-C. Appl. Surf. Sci. 2018, 435, 149.

[88] Cheon, Y. E.; Suh, M. P. Angew. Chem., Int. Ed. 2009, 48, 2899.

[89] Suh, M. P.; Moon, H. R.; Lee, E. Y.; Jang, S. Y. J. Am. Chem. Soc. 2006, 128,4710

[90] Shakya, S.; He, Y.; Ren, X.; Guo, T.; Maharjan, A.; Luo, T.; Wang, T.; Dhakhwa, R.; Regmi, B.; Li, H.; Gref, R.; Zhang, J. Small 2019 , 15, 1901065

[91] Gao, X.; Hai, X.; Baigude, H.; Guan, W.; Liu, Z. Sci. Rep. 2016, 6, 37705.

[92] Horcajada, P.; Serre, C.; Vallet-Regi, M.; Sebban, M.; Taulelle, F.; Ferey, G. Angew. Chem., Int. Ed. 2006, 45, 5974.

[93] Li, S.; Wang, K.; Shi, Y.; Cui, Y.; Chen, B.; He, B.; Dai, W.; Zhang, H.; Wang, X.; Zhong, C.; Wu, H.; Yang, Q.; Zhang, Q. Adv. Funct. Mater. 2016, 26, 2715.

[94] Guan, D.; Chen, F.; Qiu, Y.; Jiang, B.; Gong, L.; Lan, L.; Huang, W. Angew. Chem., Int. Ed. 2019, 58, 6678.

[95] Lin, S.; Liu, X.; Tan, L.; Cui, Z.; Yang, X.; Yeung, K. W. K.; Pan, H.; Wu, S. ACS Appl. Mater. Interfaces 2017, 9, 19248.
[96] Chen, H.; Yang, J.; Sun, L.; Zhang, H.; Guo, Y.; Qu, J.; Jiang, W.; Chen, W.; Ji, J.; Yang, Y.-W.; Wang, B. Small 2019, 15, 1903880.

[97] Duan, F.; Feng, X.; Jin, Y.; Liu, D.; Yang, X.; Zhou, G.; Liu, D.; Li, Z.; Liang, X.-J.; Zhang, J. Biomaterials 2017, 144, 155.

[98] Mao, D.; Hu, F.; Kenry; Ji, S.; Wu, W.; Ding, D.; Kong, D.; Liu, B. Adv. Mater. 2018, 30, 1706831.

[99] Sava Gallis, D. F.; Butler, K. S.; Agola, J. O.; Pearce, C. J.; McBride, A. A. ACS Appl. Mater. Interfaces 2019, 11, 7782.

[100] Vallabani, N. V. S.; Vinu, A.; Singh, S.; Karakoti, A. J. Colloid Interface Sci. 2020, 567, 154

[101] Xi, J.; Wei, G.; An, L.; Xu, Z.; Xu, Z.; Fan, L.; Gao, L. Nano Lett. 2019, 19, 7645 .

[102] Xi, J.; Wei, G.; Wu, Q.; Xu, Z.; Liu, Y.; Han, J.; Fan, L.; Gao, L. Biomater. Sci. 2019, 7, 4131.

[103] Ye, Y.; Xiao, L.; He, B.; Zhang, Q.; Nie, T.; Yang, X.; Wu, D.; Cheng, H.; Li, P.; Wang, Q. J. Mater. Chem. B 2017, 5, 1518.

[104] Liu, X.; Yan, Z.; Zhang, Y.; Liu, Z.; Sun, Y.; Ren, J.; Qu, X. ACS Nano 2019, 13, 5222. 